\title{
Reliable computation of phase stability and equilibrium using interval methods
}

\author{
Mark A. Stadtherr, ${ }^{*}$ Gang Xu, $\dagger$ Gabriela I. Burgos- \\ Solórzanoł and William D. Haynes§ \\ Department of Chemical and Biomolecular Engineering, University of Notre \\ Dame, Notre Dame, IN 46556 USA \\ *Corresponding Author \\ †Current address: Invensys/SimSci-Esscor, 26561 Rancho Parkway South, Suite \\ 100, Lake Forest, CA 92630 USA \\ $\ddagger$ Current address: DuPont Titanium Technologies, Rte. 141 \& Henry Clay Rd., \\ Wilmington, DE 19880 USA \\ $\S$ Current address: NFS Inc., 1205 Banner Hill Road, Erwin, TN 37650 USA
}

\begin{abstract}
The reliable calculation of phase equilibrium is a critical issue in the simulation, optimization and design of a wide variety of industrial processes, especially those involving separation operations such as distillation and extraction. However, even when accurate models of the necessary thermodynamic properties are available, it is often very difficult to actually solve the phase equilibrium problem reliably. In this paper, we will discuss a deterministic method, based on interval analysis, that provides a mathematical and computational guarantee that the phase equilibrium problem has been correctly solved, and will highlight recent results using such an approach. Cases are considered in which published computational results are shown to be incorrect.
\end{abstract}

Keywords: Phase equilibrium; Phase stability; Interval analysis; Global optimization

Reference to this paper should be made as follows: Stadtherr, M. A., Xu, G., BurgosSolórzano, G. I. and Haynes, W. D. (xxxx) 'Reliable computation of phase stability and equilibrium using interval methods,' Int. J. of Reliability and Safety, Vol. x, No. $\mathrm{x}, \mathrm{pp} \cdot \mathrm{xxx}-\mathrm{xxx}$.

Biographical notes: Mark A. Stadtherr is Professor of Chemical and Biomolecular Engineering at the University of Notre Dame. The focus of his research is primarily on the development and application of strategies for reliable engineering computing, with particular interests in global optimization, verified solution of dynamic systems, computation of phase behavior, and ecological modeling.

\section{INTRODUCTION}

In computing phase equilibrium, the goals are to correctly determine the number and type of phases present, and the distribution of components amongst the phases, at the equilibrium state. The reliable calculation of phase equilibrium for multicomponent mixtures is a critical issue in the simulation, optimization and design of a wide variety of industrial processes, especially those involving separation operations such as distillation and extraction. It is also important in the simulation of enhanced oil recovery processes such as miscible or immiscible gas flooding. However, even when accurate models of the necessary thermo- dynamic properties are available, it is often very difficult to actually solve the phase equilibrium problem reliably, an issue that may have significant implications. For example, distillation operations are typically designed with the expectation that there be exactly two phases (vapor and liquid) on each tray. The unexpected and unpredicted appearance of a second liquid phase on one or more trays could substantially change the operating characteristics of the distillation column, resulting in failure to achieve the desired separation performance, or even resulting in safety issues.

For the case of fixed temperature and pressure, which 
will be the focus here, the fundamental thermodynamic condition that must be achieved at phase equilibrium is the global minimization of the total Gibbs energy. Though easily stated in principle, in practice the computation of phase equilibrium is a very challenging problem. As a result, there is a very large and still growing body of literature devoted to the solution of this problem. As reviewed by Seider and Widagdo (1996), there have been proposed a wide variety of problem formulations and numerical solution procedures, involving both direct optimization and the solution of equivalent nonlinear equation systems. Some methods use local optimization and/or equation-solving methods, perhaps in connection with some multistart approach, or the use of homotopy-continuation. Stochastic global optimization methods (e.g., simulated annealing, genetic algorithms, etc.) have also been frequently proposed in this context. However, none of these techniques is actually guaranteed to produce the correct results, and, with very few exceptions, these methods may fail to solve the phase equilibrium problem correctly in some cases. In this paper, we will discuss a deterministic method, based on interval analysis, that provides a mathematical and computational guarantee that the phase equilibrium problem has been correctly solved, and will highlight recent results using such an approach. Cases are considered in which published computational results are shown to be incorrect (not stable phase equilibria).

\section{BACKGROUND}

For determining phase equilibrium at constant temperature and pressure, the total Gibbs energy of the system is minimized. This computation is commonly done in two (possibly alternating) stages, as outlined by Michelsen $(1982 \mathrm{a}, \mathrm{b})$. The first involves the phase stability problem; that is, to determine whether a given mixture (test phase) will split into multiple phases. The second involves the phase split problem; that is, to determine the amounts and compositions of the phases assumed to be present. Phase stability analysis may be interpreted as a global optimality test that determines whether the phase being tested corresponds to a global minimum in the total Gibbs energy of the system. If it is determined that a phase will split, then a phase split problem is solved, which can be interpreted as finding a local minimum in the total Gibbs energy. This local minimum can then be tested for global optimality using phase stability analysis. If necessary, the phase split calculation must then be repeated, perhaps changing the number of phases assumed to be present, until a solution is found that meets the global optimality test. Clearly the correct solution of the phase stability problem, itself a global optimization problem, is the key in this two-stage global optimization procedure for phase equilibrium. However, in solving the phase stability problem, conventional solution methods are initialization dependent, and may fail by converging to trivial or nonphysical solutions or to a point that is a local but not a global minimum. Thus, there has been significant interest in the development of deterministic techniques that guarantee the correct solution of the phase stability problem, as reviewed briefly below.

Accurate predictions of phase equilibrium also require a good model of the Gibbs energy of the system, in terms of the system temperature, pressure and composition. This is usually based on an excess Gibbs energy model (activity coefficient model) and/or an equation-of-state (EOS) model. When the same model is used to represent all phases, the overall model is referred to as symmetric. When different models are used to represent different types of phases, the overall model is referred to as asymmetric.

One approach for deterministic phase stability analysis, as demonstrated by McDonald and Floudas (1995a,b,c, 1997) for symmetric cases in which various excess Gibbs energy models were used, is the use of deterministic global optimization techniques, such as GOP (Floudas and Visweswaran, 1990, 1993) and branch-and-bound (Falk and Soland, 1969). McDonald and Floudas (1995a,b,c, 1997) also considered the asymmetric case in which an excess Gibbs energy model was used for liquid phases, and the vapor was an ideal gas. A more general branch-andbound strategy, the $\alpha$-BB method, was applied by Harding and Floudas (2000) to symmetric cases in which cubic EOS models were used. The $\alpha$-BB approach relies on the use of convex underestimating functions to obtain lower bounds on the objective function. However, whether these are rigorously valid bounds depends on the proper choice of a parameter $(\alpha)$. Methods exist (Adjiman et al., 1998), based on an interval representation of the Hessian matrix, that can be used to guarantee a proper value of $\alpha$, and this approach was applied by Harding and Floudas (2000).

An alternative deterministic procedure for phase stability analysis is the use of an interval-Newton approach (Schnepper and Stadtherr, 1996). This approach was first demonstrated for symmetric cases using excess Gibbs energy models by Stadtherr et al. (1995), and later by McKinnon et al. (1996) and Tessier et al. (2000), and was first demonstrated for symmetric cases using cubic EOS models by Hua et al. (1996a,b, 1998). Xu et al. (2002) also applied this approach to the symmetric case in which a statistical associating fluid theory (SAFT) model is used. The interval-Newton procedure provides a mathematical guarantee that the phase stability problem is correctly solved. Moreover, since it uses interval arithmetic throughout, and thus bounds rounding error, the interval-Newton method also provides a rigorous computational guarantee of global optimality (Hansen and Walster, 2004). We highlight here new and recent results using this approach, on both symmetric and asymmetric models, focusing on cases in which published computational results are shown to be incorrect.

\section{PHASE STABILITY ANALYSIS}

The determination of phase stability is typically done using tangent plane analysis, based on the test formulated by Baker et al. (1982). Consider a system with $n$ components, 


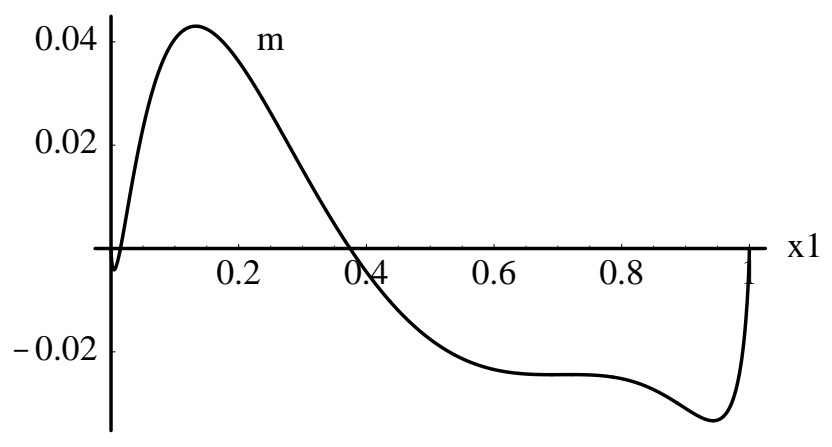

Figure 1: Example of a reduced Gibbs energy surface, here expressed as the Gibbs energy of mixing $m$ vs. mole fraction $x_{1}$

and assume that the phase to be tested has a composition (mole fraction) vector $\boldsymbol{x}_{0}$ and that a constant temperature $T$ and pressure $P$ have been specified. Then consider the molar reduced Gibbs energy vs. composition (mole fraction) surface $g(\boldsymbol{x})$ and a hyperplane tangent to $g(\boldsymbol{x})$ at $\boldsymbol{x}=\boldsymbol{x}_{0}$. If this tangent plane ever crosses (goes above) the Gibbs energy surface $g(\boldsymbol{x})$, then the phase being tested is not stable (i.e., it is either unstable or metastable). This condition is often stated in terms of the tangent plane distance (TPD) function $D(\boldsymbol{x})$ that gives the distance of the Gibbs energy surface above the tangent plane. This is given by

$$
D(\boldsymbol{x})=g(\boldsymbol{x})-g_{0}-\boldsymbol{s}_{0}^{\mathrm{T}}\left(\boldsymbol{x}-\boldsymbol{x}_{0}\right),
$$

where $g_{0}=g\left(\boldsymbol{x}_{0}\right)$ and $\boldsymbol{s}_{0}=\nabla g\left(\boldsymbol{x}_{0}\right)$ are the reduced Gibbs energy function and its gradient evaluated at the feed composition $\boldsymbol{x}_{0}$. If $D(\boldsymbol{x})$ is negative for any value of $\boldsymbol{x}$, then the phase being tested is not stable.

To illustrate the use of the tangent plane criterion, consider the binary $(n=2) g(\boldsymbol{x})$ curve given in Fig. 1. Here $g(\boldsymbol{x})$ is expressed relative to a reference state consisting of the pure components at system temperature and pressure and is labeled $m$, the reduced Gibbs energy of mixing. For a test phase (feed) of composition $\boldsymbol{x}_{0}=(0.95,0.05)^{\mathrm{T}}$, the situation is shown in Fig. 2. Here it is seen that the tangent $\left(m_{\tan }\right)$ to the Gibbs energy of mixing curve $m$ at $x_{0,1}=0.95$ never crosses the Gibbs energy curve $m$. Thus, $D$ is never negative, and this test phase is stable. On the other hand, Fig. 3 shows the situation for a feed of composition $\boldsymbol{x}_{0}=(0.65,0.35)^{\mathrm{T}}$. Here it is seen that the tangent $\left(m_{\mathrm{tan}}\right)$ to the Gibbs energy of mixing curve $m$ at $x_{0,1}=0.65$ does cross the Gibbs energy curve $m$. Thus, $D$ becomes negative, and this test phase is not stable.

Computationally, to determine if $D$ is ever negative, its

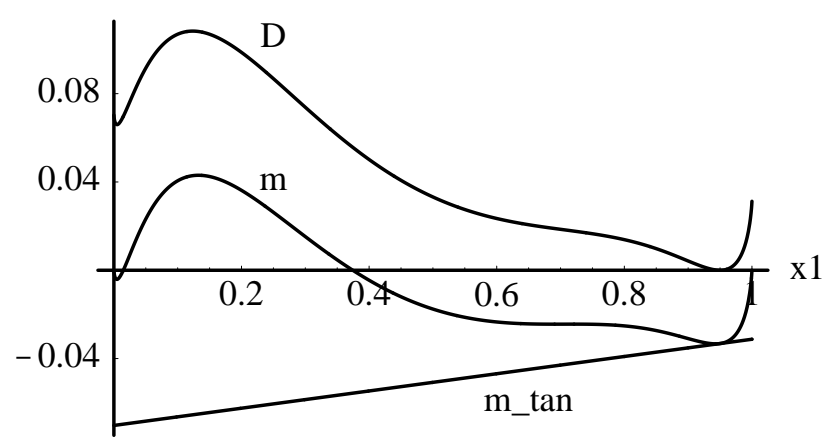

Figure 2: Tangent plane analysis for feed composition of $x_{0,1}=0.95$. This feed is stable.

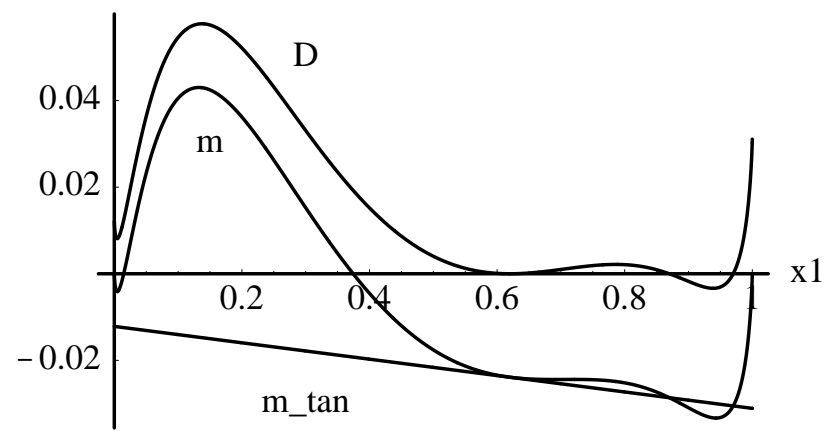

Figure 3: Tangent plane analysis for feed composition of $x_{0,1}=0.65$. This feed is not stable.

minimum is sought by solving an optimization problem:

$$
\begin{array}{ll} 
& \min _{\boldsymbol{x}} D(\boldsymbol{x}) \\
\text { s.t. } & 1-\sum_{i=1}^{n} x_{i}=0 .
\end{array}
$$

If a stationary point (local minimum) of $D$ is found for which $D<0$, then this indicates that the phase being tested is not stable. Actually, to show that a phase is not stable, it is sufficient to find any point $\boldsymbol{x}$ for which $D<0$. However, stationary points with $D<0$ are commonly sought since they are useful in providing initial composition estimates for a possible new equilibrium phase or phases, and thus are useful in solving the phase split problem. Proof that the phase being tested is stable is obtained if the global minimum of $\mathrm{D}$ is zero (corresponding to the tangent point at the feed composition $\boldsymbol{x}_{0}$ ). Obviously this procedure may fail if the global minimum of the tangent plane distance function is not found. For instance, if the optimization algorithm used returns a global minimum of zero, while the true global minimum is negative, the conclusion that the phase is stable will be incorrect. 


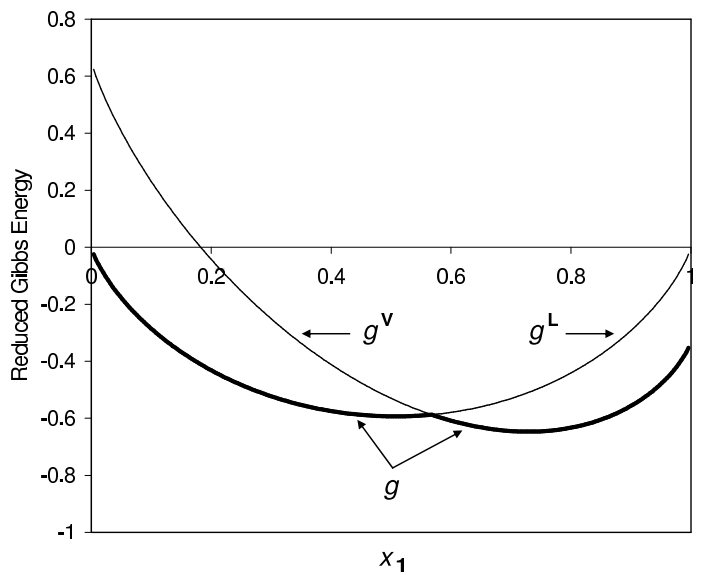

Figure 4: Example of a reduced Gibbs energy surface for an asymmetric model.

The foregoing assumes that the overall Gibbs energy model is symmetric, and that $g$ depends only on the composition $\boldsymbol{x}$, as in the case of excess Gibbs energy models. If an EOS model is used, again considering only the symmetric case, then the optimization problem to be solved is:

$$
\begin{array}{ll} 
& \min _{\boldsymbol{x}, v} D(\boldsymbol{x}, v) \\
\text { s.t. } & 1-\sum_{i=1}^{n} x_{i}=0 \\
& f(\boldsymbol{x}, v)=0 .
\end{array}
$$

Here $v$ is the molar volume, which is related to the composition $\boldsymbol{x}$ by the EOS $f(\boldsymbol{x}, v)=0$. EOS models may also be written in terms of the compressibility factor $Z=P v / R T$, instead of $v$. A complication that arises when an EOS model is used is that for a given composition $\boldsymbol{x}$, there may be multiple solutions for $v$. This means that $g(\boldsymbol{x}, v)$, and thus $D(\boldsymbol{x}, v)$, may be multivalued for some values of $\boldsymbol{x}$. This situation can be avoided by using a different formulation of the problem, as described by Nagarajan et al. (1991). However, when interval methods are used, as described below, the occurrence of multiple volume roots does not cause any difficulties.

Finally we consider the asymmetric case, focusing on the case in which only vapor and liquid phases are possible. The Gibbs energy model for the liquid phase is denoted $g^{\mathrm{L}}(\boldsymbol{x})$ and is assumed to be an excess Gibbs energy model. The Gibbs energy model for the vapor phase is denoted $g^{\mathrm{V}}(\boldsymbol{x}, v)$ and is assumed to be an EOS model. In tangent plane analysis for phase stability, since the goal in testing a phase is to detect alternate states that have a lower Gibbs energy, the Gibbs energy surface that must be used is given by whichever of $g^{\mathrm{L}}(\boldsymbol{x})$ and $g^{\mathrm{V}}(\boldsymbol{x}, v)$ is lowest. That is, in Eq. (1), $g(\boldsymbol{x})=\min \left[g^{\mathrm{V}}(\boldsymbol{x}, v), g^{\mathrm{L}}(\boldsymbol{x})\right]$, as depicted in Fig. 4, and evaluations at $\boldsymbol{x}_{0}$ must be done on the lower of the Gibbs energy surfaces. It follows that $D(\boldsymbol{x})=\min \left[D^{\mathrm{V}}(\boldsymbol{x}, v), D^{\mathrm{L}}(\boldsymbol{x})\right]$, where the vapor and liquid tangent plane distance functions, $D^{\mathrm{V}}(\boldsymbol{x}, v)=g^{\mathrm{V}}(\boldsymbol{x}, v)-$

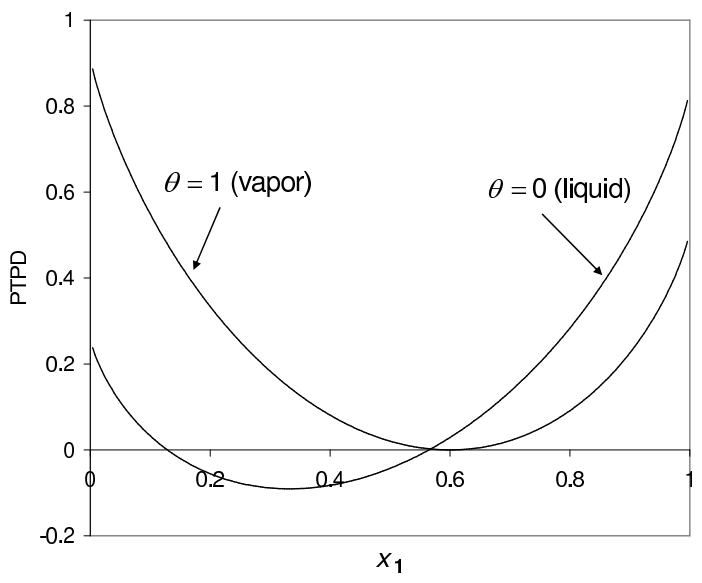

Figure 5: Pseudo tangent place distance (PTPD) function for feed composition $x_{0,1}=0.6$. This phase is not stable.

$g_{0}-\boldsymbol{s}_{0}^{\mathrm{T}}\left(\boldsymbol{x}-\boldsymbol{x}_{0}\right)$ and $D^{\mathrm{L}}(\boldsymbol{x})=g^{\mathrm{L}}(\boldsymbol{x})-g_{0}-\boldsymbol{s}_{0}^{\mathrm{T}}\left(\boldsymbol{x}-\boldsymbol{x}_{0}\right)$, respectively, are both based on the same values of $g_{0}$ and $\boldsymbol{s}_{0}$, as determined from whichever Gibbs energy surface is lower at $\boldsymbol{x}_{0}$. The minimization problem that must be solved is

$$
\begin{gathered}
\min _{\boldsymbol{x}, v}\left\{\min \left[D^{\mathrm{V}}(\boldsymbol{x}, v), D^{\mathrm{L}}(\boldsymbol{x})\right]\right\} \\
\text { s.t. } \quad 1-\sum_{i=1}^{n} x_{i}=0 \\
f(\boldsymbol{x}, v)=0 .
\end{gathered}
$$

To avoid the difficulties associated with the nondifferentiable objective function, it is convenient to reformulate the problem, as originally described by Xu et al. (2005). This involves use of a "pseudo tangent plane distance" (PTDP) function

$$
\widetilde{D}(\boldsymbol{x}, v, \theta)=\theta D^{\mathrm{V}}(\boldsymbol{x}, v)+(1-\theta) D^{\mathrm{L}}(\boldsymbol{x}),
$$

where $\theta$ is a binary variable whose value is determined as part of the optimization problem. For example, for the Gibbs energy model shown in Fig. 4, with feed composition $\boldsymbol{x}=(0.60,0.40)^{\mathrm{T}}$, the corresponding pseudo tangent plane distance function is shown in Fig. 5. The minimization problem that must be solved can now be expressed as:

$$
\begin{gathered}
\min _{\boldsymbol{x}, v, \theta} \widetilde{D}(\boldsymbol{x}, v, \theta) \\
\text { s.t. } \quad 1-\sum_{i=1}^{n} x_{i}=0 \\
f(\boldsymbol{x}, v)=0 \\
\theta(1-\theta)=0 .
\end{gathered}
$$

Note that the last equation is equivalent to $\theta \in\{0,1\}$. While $\theta$ appears here as a continuous variable, it will be treated explicitly as a binary variable when this system is solved, as explained below.

We now consider a method that can be used to obtain, with mathematical and computational certainty, the global minimum in the optimization problem that must be solved to determine phase stability. 


\section{SOLUTION METHOD}

\subsection{Interval analysis}

A real interval $X$ is defined as the set of real numbers lying between (and including) given upper and lower bounds; that is, $X=[\underline{X}, \bar{X}]=\{x \in \Re \mid \underline{X} \leq x \leq \bar{X}\}$. Here an underline is used to indicate the lower bound of an interval, and an overline is used to indicate the upper bound. A real interval vector $\boldsymbol{X}=\left(X_{1}, X_{2}, \ldots, X_{n}\right)^{\mathrm{T}}$ has $n$ real interval components and can be interpreted geometrically as an $n$-dimensional rectangle or box. In this context uppercase quantities are intervals, and lowercase quantities or uppercase quantities with underline or overline are real numbers.

Basic arithmetic operations with intervals are defined by $X$ op $Y=\{x$ op $y \mid x \in X, y \in Y\}$, where op $\in$ $\{+,-, \times, \div\}$. Interval versions of the elementary functions can be similarly defined. When machine computations with interval arithmetic operations are done, as in the procedures outlined below, the endpoints of an interval are computed with a directed (outward) rounding. That is, the lower endpoint is rounded down to the next machine-representable number, and the upper endpoint is rounded up to the next machine-representable number. In this way, through the use of interval arithmetic, as opposed to floating-point arithmetic, any potential rounding error problems are avoided. Several good introductions to interval analysis, as well as interval arithmetic and other aspects of computing with intervals, are available (e.g., Hansen and Walster, 2004; Jaulin et al., 2001; Kearfott, 1996; Moore, 1966; Neumaier, 1990). Implementations of interval arithmetic and elementary functions are also readily available, and recent compilers from Sun Microsystems directly support interval arithmetic and an interval data type.

For an arbitrary function $f(\boldsymbol{x})$, the interval extension $F(\boldsymbol{X})$ encloses all possible values of $f(\boldsymbol{x})$ for $\boldsymbol{x} \in \boldsymbol{X}$; that is, it encloses the range of $f(\boldsymbol{x})$ over $\boldsymbol{X}$. It is often computed by substituting the given interval $\boldsymbol{X}$ into the function $f(\boldsymbol{x})$ and then evaluating the function using interval arithmetic. This "natural" interval extension is often wider than the actual range of function values, although it always includes the actual range. For example, the natural interval extension of $f(x)=x /(x-1)$ over the interval $X=$ $[2,3]$ is $F([2,3])=[2,3] /([2,3]-1)=[2,3] /[1,2]=[1,3]$, while the true function range over this interval is $[1.5,2]$. This overestimation of the function range is due to the "dependency" problem, which may arise when a variable occurs more than once in a function expression. While a variable may take on any value within its interval, it must take on the same value each time it occurs in an expression. However, this type of dependency is not recognized when the natural interval extension is computed. In effect, when the natural interval extension is used, the range computed for the function is the range that would occur if each instance of a particular variable were allowed to take on a different value in its interval range. For the case in which $f(\boldsymbol{x})$ is a single-use expression, that is, an expression in which each variable occurs only once, natural interval arithmetic always yields the true function range. For example, rearrangement of the function expression used above gives $f(x)=x /(x-1)=1+1 /(x-1)$, and now $F([2,3])=$ $1+1 /([2,3]-1)=1+1 /[1,2]=1+[0.5,1]=[1.5,2]$, the true range. For cases in which such rearrangements are not possible, there are a variety of other approaches that can be used to try to tighten interval extensions (Hansen and Walster, 2004; Jaulin et al., 2001; Kearfott, 1996; Makino and Berz, 1999, 2003; Neumaier, 1990, 2003).

\subsection{Interval-Newton method}

Of particular interest here is the interval-Newton method, an outline of which is given here. More details are available elsewhere (e.g., Hansen and Walster, 2004; Kearfott, 1996). The interval-Newton method is an equation-solving method. In the context of phase stability, it is used to seek solutions of the first-order optimality conditions corresponding to the minimization problems described above. The global minimum in the optimization problem will be one of these stationary points. This technique is not equivalent to simply implementing the routine "point" Newton method in interval arithmetic.

Consider the solution of the general nonlinear equation system $\boldsymbol{f}(\boldsymbol{x})=\mathbf{0}$, which in this context represents the firstorder optimality conditions associated with minimizing an objective function $\phi(\boldsymbol{x})$. Given some initial search interval, $\boldsymbol{X}^{(0)}$, the interval-Newton algorithm is applied to a sequence of subintervals. For a subinterval $\boldsymbol{X}^{(k)}$ in the sequence (beginning with $k=0$ ), the first step is the function range test. An interval extension $\boldsymbol{F}\left(\boldsymbol{X}^{(k)}\right)$ of the function $\boldsymbol{f}(\boldsymbol{x})$ is calculated. If there is any component of the interval extension $\boldsymbol{F}\left(\boldsymbol{X}^{(k)}\right)$ that does not include zero, then no solution of $\boldsymbol{f}(\boldsymbol{x})=\mathbf{0}$, can exist in this interval. This interval can then be discarded since the global minimum must be one of the stationary points. The next subinterval in the sequence may then be considered. Otherwise, testing of $\boldsymbol{X}^{(k)}$ continues. During this step, other intervalbased techniques (e.g., constraint propagation) may also be applied to try to shrink $\boldsymbol{X}^{(k)}$ before proceeding.

The next step is the objective range test. An interval extension $\Phi\left(\boldsymbol{X}^{(k)}\right)$, containing the range of $\phi(\boldsymbol{x})$ over $\boldsymbol{X}^{(k)}$ is computed. If the lower bound of $\Phi\left(\boldsymbol{X}^{(k)}\right)$ is greater than a known upper bound $\widehat{\phi}$ on the global minimum, then $\boldsymbol{X}^{(k)}$ can be discarded since it cannot contain the global minimum and need not be further tested. Frequently, in phase stability analysis, this test will be turned off, since it is desired to obtain all the stationary points, not just the global minimum. The stationary points are useful to initialize the solution of the phase split problem when a phase is found to be not stable.

If $\boldsymbol{X}^{(k)}$ has not been eliminated in the objective range test, then the next step is the interval-Newton test. The linear interval equation system

$$
\boldsymbol{F}^{\prime}\left(\boldsymbol{X}^{(k)}\right)\left(\boldsymbol{N}^{(k)}-\widetilde{\boldsymbol{x}}^{(k)}\right)=-\boldsymbol{f}\left(\widetilde{\boldsymbol{x}}^{(k)}\right),
$$


is solved for a new interval $\boldsymbol{N}^{(k)}$, where $\boldsymbol{F}^{\prime}\left(\boldsymbol{X}^{(k)}\right)$ is an interval extension of the Jacobian of $\boldsymbol{f}(\boldsymbol{x})$, and $\widetilde{\boldsymbol{x}}^{(k)}$ is an arbitrary point in $\boldsymbol{X}^{(k)}$. It has been shown (e.g., Hansen and Walster, 2004; Kearfott, 1996; Neumaier, 1990) that any root of $\boldsymbol{f}(\boldsymbol{x})=\mathbf{0}$ contained in $\boldsymbol{X}^{(k)}$ is also contained in the image $\boldsymbol{N}^{(k)}$. This implies that if the intersection between $\boldsymbol{X}^{(k)}$ and $\boldsymbol{N}^{(k)}$ is empty, then no root exists in $\boldsymbol{X}^{(k)}$, and also suggests the iteration scheme $\boldsymbol{X}^{(k+1)}=\boldsymbol{X}^{(k)} \cap \boldsymbol{N}^{(k)}$. In addition, it has also been shown (e.g., Hansen and Walster, 2004; Kearfott, 1996; Neumaier, 1990) that, if $\boldsymbol{N}^{(k)} \subset \boldsymbol{X}^{(k)}$, then there is a unique root contained in $\boldsymbol{X}^{(k)}$ and thus in $\boldsymbol{N}^{(k)}$. Thus, after computation of $\boldsymbol{N}^{(k)}$ from Eq. (7), there are three possibilities: (1) $\boldsymbol{X}^{(k)} \cap \boldsymbol{N}^{(k)}=\varnothing$, meaning there is no root in the current interval $\boldsymbol{X}^{(k)}$, and it can be discarded; (2) $\boldsymbol{N}^{(k)} \subset \boldsymbol{X}^{(k)}$, meaning that there is exactly one root in the current interval $\boldsymbol{X}^{(k)}$; and (3) neither of the above, meaning that no conclusion can be drawn. In the last case, if $\boldsymbol{X}^{(k)} \cap \boldsymbol{N}^{(k)}$ is sufficiently smaller than $\boldsymbol{X}^{(k)}$, then the interval-Newton test can be reapplied to the resulting intersection, $\boldsymbol{X}^{(k+1)}=\boldsymbol{X}^{(k)} \cap \boldsymbol{N}^{(k)}$. Otherwise, the intersection $\boldsymbol{X}^{(k)} \cap \boldsymbol{N}^{(k)}$ is bisected, and the resulting two subintervals are added to the sequence of subintervals to be tested. If an interval containing a unique root has been identified, then this root can be tightly enclosed by continuing the interval-Newton iteration, which will converge quadratically to a desired tolerance (on the enclosure diameter).

Clearly, the solution of the linear interval system given by Eq. (7) is essential to this approach. To see the issues involved in solving such a system, consider the general linear interval system $\boldsymbol{A} \boldsymbol{z}=\boldsymbol{B}$, where the matrix $\boldsymbol{A}$ and the right-hand-side vector $\boldsymbol{B}$ are intervalvalued. The solution set $\mathcal{S}$ of this system is defined by $\mathcal{S}=\{\boldsymbol{z} \mid \widetilde{A} \boldsymbol{z}=\boldsymbol{b}, \widetilde{A} \in \boldsymbol{A}, \boldsymbol{b} \in \boldsymbol{B}\}$. However, in general this set is not an interval and may have a very complex, polygonal geometry. Thus to "solve" the linear interval system, one instead seeks an interval $\boldsymbol{Z}$ containing $\mathcal{S}$. Computing the interval hull (the tightest interval containing $\mathcal{S}$ ) is NP-hard (Rohn and Kreinovich, 1995), but there are several methods for determining an interval $\boldsymbol{Z}$ that contains but overestimates $\mathcal{S}$. Various interval-Newton methods differ in how they solve Eq. (7) for $\boldsymbol{N}^{(k)}$ and thus in the tightness with which the solution set is enclosed. By obtaining bounds that are as tight as possible, the overall performance of the interval-Newton approach can be improved, since with a smaller $\boldsymbol{N}^{(k)}$ the volume of $\boldsymbol{X}^{(k)} \cap \boldsymbol{N}^{(k)}$ is reduced, and it is also more likely that either $\boldsymbol{X}^{(k)} \cap \boldsymbol{N}^{(k)}=\varnothing$ or $\boldsymbol{N}^{(k)} \subset \boldsymbol{X}^{(k)}$ will be satisfied. Thus, intervals that may contain solutions of the nonlinear system are more quickly contracted, and intervals that contain no solution or that contain a unique solution may be more quickly identified, all of which leads to a likely reduction in the number of bisections needed.

Frequently, $\boldsymbol{N}^{(k)}$ is computed component-wise using an interval Gauss-Seidel approach, preconditioned with an approximate inverse-midpoint matrix. Though the inversemidpoint preconditioner is a good general-purpose precon- ditioner, it is not always the most effective approach (Kearfott, 1996). Recently, a hybrid preconditioning approach (HP/RP), which combines a simple pivoting preconditioner (and a method for selecting the real point $\widetilde{\boldsymbol{x}}^{(k)}$ ) with the standard inverse-midpoint scheme, has been described by Gau and Stadtherr (2002) and shown to achieve substantially more efficient computational performance than the inverse-midpoint preconditioner alone, in some cases by multiple orders of magnitude. However, it still cannot yield the tightest enclosure of the solution set, which, as noted above, is in general an NP-hard problem. Lin and Stadtherr (2004a,b) have recently suggested a strategy (LISS_LP), based on linear programming (LP), for solving the linear interval system, Eq. (7), arising in the context of interval-Newton methods. Using this approach, exact component-wise bounds on the solution set can be calculated, while avoiding exponential time complexity. In numerical experiments (Lin and Stadtherr, 2004a,b), LISS_LP has been shown to achieve further computational performance improvements compared with HP/RP.

This approach, as outlined above, is referred to as an interval-Newton/generalized-bisection (IN/GB) method. An important feature of this approach is that, unlike standard methods for nonlinear equation solving and/or optimization that require a point initialization, the IN/GB methodology requires only an initial interval, and this interval can be sufficiently large to enclose all feasible results. Thus, in the case of phase stability analysis, all composition variables (mole fractions) $x_{i}$ can be initialized to the interval $[0,1]$. For the vapor-phase molar volume $v$, an initial interval corresponding to a compressibility factor $(Z=P v / R T)$ interval of $[0.5,1]$ is used. The lower limit of 0.5 effectively eliminates any liquid-like volume roots (the EOS model is used to represent the vapor phase only) and in most circumstances is reasonable for the relatively low pressures at which asymmetric models are typically used. If the pressure was so high that the vapor-phase compressibility was less than 0.5 , then a symmetric model using an EOS for both vapor and liquid phases would probably be used. Of course, the initial interval for $v$ can easily be modified as desired to fit other circumstances. Finally, for the binary variable $\theta$, the initial interval is set to $[0,1]$. For this binary variable, a special bisection rule is used. If $\theta$ is chosen by the IN/GB algorithm as the variable to be bisected, then it is bisected into the degenerate (thin) intervals $[0,0]$ and $[1,1]$. Thus $\theta$ can be bisected only once. In this way, $\theta$ is treated explicitly as a binary (rather than continuous) variable in solving the equation system.

On completion, if the objective range test has been turned off, the IN/GB algorithm will have determined, with certainty, narrow enclosures of all the stationary points of $D$, including local and global optima, and thus the global minimum can be readily determined. Alternatively, if the objective range test has been turned on, the IN/GB scheme will lead directly to the global minimum without finding any of the other stationary points. However, as noted above, if the tested phase is not stable, knowledge of the stationary points can be useful for 
initializing phase split computations.

\section{EXAMPLES}

\section{$5.1 \quad$ Example 1}

In this example, we consider an asymmetric model of the binary mixture 2,3-dimethyl-2-butane (component 1 ) and methanol (component 2). The liquid phase is modeled using the NRTL excess Gibbs energy model and the vapor phase using the Soave-Redlich-Kwong (SRK) EOS model. Thus, for the liquid phase,

$$
g^{\mathrm{L}}(\boldsymbol{x})=\sum_{i=1}^{n} x_{i} \ln x_{i}+\sum_{i=1}^{n} x_{i} \frac{\sum_{j=1}^{n} x_{j} \tau_{j i} G_{j i}}{\sum_{k=1}^{n} x_{k} G_{k i}},
$$

where $G_{i j}=\exp \left(-\alpha_{i j} \tau_{i j}\right)$ and $\tau_{i j}=A_{i j} / R T$. Values of the parameters $\alpha$ and $A_{i j}$ are given below. This reduced Gibbs energy function is expressed relative to the pure components as liquids at system temperature and pressure. The reduced Gibbs energy for the vapor phase, relative to the same reference state is

$$
\begin{aligned}
g^{\mathrm{V}}(\boldsymbol{x}, v) & =\sum_{i=1}^{n} x_{i} \ln x_{i}+\sum_{i=1}^{n} x_{i} \ln \breve{\phi}_{i}(\boldsymbol{x}, v) \\
- & \sum_{i=1}^{n} x_{i}\left[v_{i}^{\mathrm{L}}\left(P-P_{i}^{\mathrm{sat}}\right)+\ln \phi_{i}^{\mathrm{sat}}+\ln \frac{P_{i}^{\mathrm{sat}}}{P}\right] .
\end{aligned}
$$

Here $\breve{\phi}_{i}(\boldsymbol{x}, v)$ is the fugacity coefficient of component $i$ in the mixture at system $T$ and $P, v_{i}^{\mathrm{L}}$ is the molar volume of pure $i$ as a liquid at system $T$ (assumed independent of pressure and evaluated at saturation), $P_{i}^{\text {sat }}$ is the vapor pressure of pure $i$ at system $T$, and $\phi_{i}^{\text {sat }}$ is the fugacity coefficient of pure $i$ as a vapor at $P_{i}^{\text {sat }}$ and system $T$. The SRK EOS is given by

$$
f(\boldsymbol{x}, v)=P-\frac{R T}{v-b}+\frac{a}{v(v+b)}=0 .
$$

Here $a$ and $b$ are functions of $\boldsymbol{x}$ determined by specified mixing rules. The "standard" mixing rules are $b=\sum_{i=1}^{n} x_{i} b_{i}$ and $a=\sum_{i=1}^{n} \sum_{j=1}^{n} x_{i} x_{j} a_{i j}$, with $a_{i j}=$ $\left(1-k_{i j}\right) \sqrt{a_{i} a_{j}}$. The $a_{i}$ and $b_{i}$ are pure component properties determined from the system temperature $T$, the critical temperatures $T_{c i}$, the critical pressures $P_{c i}$ and acentric factors $\omega_{i}$ according to

$$
\begin{gathered}
b_{i}=0.08664 \frac{R T_{c i}}{P_{c i}} \\
a_{i}=0.42748 \alpha \frac{R^{2} T_{c i}^{2}}{P_{c i}} \\
\alpha=\left[1+\left(0.480+1.574 \omega_{i}-0.176 \omega_{i}^{2}\right)\left(1-\sqrt{T / T_{c i}}\right)\right]^{2} .
\end{gathered}
$$

The binary interaction parameter $k_{i j}$ is generally determined experimentally by fitting binary vapor-liquid equilibrium data. Values of the model parameters are given below. The mixture fugacity coefficients $\breve{\phi}_{i}(\boldsymbol{x}, v)$ are determined (Prausnitz et al., 1986) from the SRK EOS model, using the relationship

$$
\begin{aligned}
\ln \breve{\phi}_{i}(\boldsymbol{x}, v)= & \ln \frac{v}{v-b}+\frac{b_{i}}{v-b}-\frac{2 \sum_{j=1}^{n} x_{j} a_{j i}}{R T b} \ln \frac{v+b}{v} \\
& +\frac{a b_{i}}{R T b^{2}}\left[\ln \frac{v+b}{v}-\frac{b}{v+b}\right]-\ln \frac{P v}{R T} .
\end{aligned}
$$

The equation of state can also be used to determine $\phi_{i}^{\text {sat }}$. However, since $P_{i}^{\text {sat }}$ is likely to be relatively small, it is often reasonable to assume that $\phi_{i}^{\text {sat }}=1$, and that is what is done here.

The optimization problem to be solved for this asymmetric model is given by Eq. (6). For this problem, the first-order optimality conditions, i.e., the equations solved using the interval-Newton approach, are

$$
\begin{aligned}
\frac{\partial \widetilde{D}}{\partial x_{i}}-\frac{\partial \widetilde{D}}{\partial x_{n}}=0, \quad i & =1, \ldots, n-1 \\
1-\sum_{i=1}^{n} x_{i} & =0 \\
f(\boldsymbol{x}, v) & =0 \\
\theta(1-\theta) & =0 .
\end{aligned}
$$

Experimental vapor-liquid equilibrium measurements were made for this system recently by Uusi-Kyyny et al. (2004) at atmospheric pressure. The experimental pressure varied slightly, but for most measurements was $P=101.2$ $\mathrm{kPa}$, and this is the value used here. The experimental results were modeled by Uusi-Kyyny et al. (2004) using SRK as the vapor-phase model, and using NRTL, Wilson, and UNIQUAC as different liquid-phase models. Parameters in each of the liquid-phase models were estimated by minimizing the sum of the absolute values of the relative errors between the measured activity coefficient and the activity coefficient calculated from the model. To then test the liquid-phase models, Uusi-Kyyny et al. (2004) used them to perform bubble-point calculations at each of the liquid-phase compositions $x_{1}$ on the experimental vaporliquid envelope. Comparing the computed results for the vapor-phase compositions $y_{1}$ and temperature $T$ to the experimental values showed that the average errors were $\Delta y_{1}=0.0059$ and $\Delta T=0.14 \mathrm{~K}$ for the Wilson equation, $\Delta y_{1}=0.0124$ and $\Delta T=0.41 \mathrm{~K}$ for NRTL, and $\Delta y_{1}=0.0194$ and $\Delta T=0.56 \mathrm{~K}$ for UNIQUAC. Hence, the Wilson equation apparently provided the best fit, followed by NRTL, and then UNIQUAC. We will use the NRTL model here, with the parameters $A_{12} / R=691.87$ $\mathrm{K}$ and $A_{21} / R=513.14 \mathrm{~K}\left(\alpha_{12}=\alpha_{21}=0.4\right)$, which are the values determined by Uusi-Kyyny et al. (2004). For SRK, the pure component properties used are $T_{c 1}=524.0 \mathrm{~K}$, $P_{c 1}=3160 \mathrm{kPa}, \omega_{1}=0.2333, T_{c 2}=512.6 \mathrm{~K}, P_{c 2}=8096$ $\mathrm{kPa}$, and $\omega_{2}=0.5656$, and the binary interaction parameter used is $k_{12}=0$. Pure component vapor pressures come from the Antoine equation (with $P_{i}^{\text {sat }}$ in $\mathrm{MPa}$ and $T$ in $\mathrm{K}$ ),

$$
P_{i}^{\text {sat }}=\exp \left(A_{i}-\frac{B_{i}}{T+C_{i}}\right),
$$


with $A_{1}=6.574, B_{1}=2500.8, C_{1}=-64.19, A_{2}=9.5334$, $B_{2}=3550.3$ and $C_{2}=-37.353$. The liquid molar volumes used are $v_{1}^{\mathrm{L}}=119.643 \mathrm{~cm}^{3} / \mathrm{mol}$ and $v_{2}^{\mathrm{L}}=40.7 \mathrm{~cm}^{3} / \mathrm{mol}$. All the model parameters used here are exactly as given by Uusi-Kyyny et al. (2004).

As a test problem for the phase stability analysis procedure described above, we first chose a feed composition on the experimental vapor-liquid envelope, namely $x_{0,1}=0.6233$ and $T=325.62 \mathrm{~K}$. Our expectation was that this feed would either be just slightly outside the phase envelope predicted by NRTL/SRK, in which case the feed would test as stable, or it would be just slightly inside the phase envelope, in which case the feed would test as unstable, with one stationary point near the experimental vapor-phase composition $\left(y_{1}=0.4758\right)$ showing a negative value of PTPD. However, what we actually computed for this feed composition was quite different, as shown for the first feed listed in Table 1. This feed tested as not stable, but in addition to a stationary point with negative PTPD near the experimental vapor-phase composition, there were also two other stationary points with negative PTPD. Using NRTL/SRK to do a bubble-point calculation (phase split calculation) for this liquid-phase composition $\left(x_{1}=0.6233\right)$ shows that there is a solution to the bubble-point problem at $y_{1}=0.4684$ and $T=325.243$ $\mathrm{K}$. This is very near the experimental values of $0.4758\left(\Delta y_{1}\right.$ $=0.0074)$ and $325.62 \mathrm{~K}(\Delta T=0.377 \mathrm{~K})$, and so this is the solution obtained by Uusi-Kyyny et al. (2004). Now testing this result for phase stability, with the results shown as the second feed in Table 1, as well as in the PTPD plot in Fig. 6, shows that this solution to the bubble point problem is in fact not stable. While this may be a mathematically correct solution to the bubble-point problem, it is not thermodynamically correct, and it is not a point on the vapor-liquid envelope predicted by NRTL/SRK, as was believed by Uusi-Kyyny et al. (2004). In fact, a phase split calculation, followed by phase stability analysis of the results (third feed in Table 1), shows that for a feed of $x_{0,1}$ $=0.6233$ at $T=325.243 \mathrm{~K}$, NRTL/SRK actually predicts liquid-liquid equilibrium, with one liquid phase of composition $x_{1}=0.29703$ and another liquid phase with composition $x_{1}=0.85822$ (phases in equilibrium share the same tangent plane). For each feed tested in Table 1, the computation time was less than $0.05 \mathrm{~s}$ (Pentium $43.2 \mathrm{GHz}$ ).

In Figure 7, we show the entire phase diagram for this system when calculated from Uusi-Kyyny et al. (2004)'s SRK/NRTL model, along with the experimental phase equilibrium data. These calculations were validated by using the methodology for phase stability analysis described above. Clearly the phase diagram calculated from the model does not closely match the experimental data. Experimentally, a homogeneous azeotrope is observed. However, the model predicts a heterogeneous azeotrope, i.e. a vapor-liquid-liquid equilibrium (VLLE) line. The model predicts that there should be no liquid phases observed with compositions in the range from roughly $x_{1}=0.298$ to $x_{1}=0.858$ (end points on the VLLE line), but in fact there

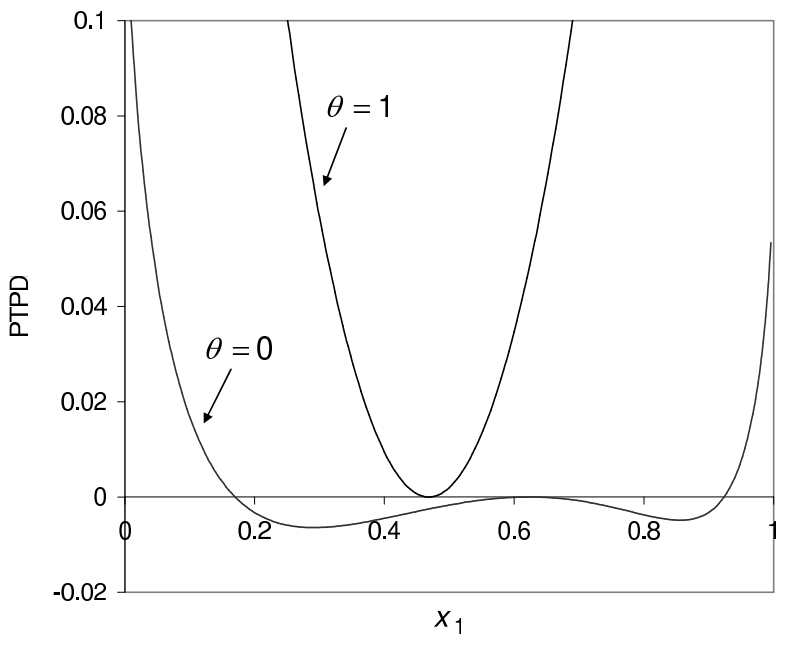

Figure 6: Plot of pseudo tangent plane distance (PTPD) function in Example 1 for vapor-liquid equilibrium computed by Uusi-Kyyny et al. (2004). This is not a stable state. See text for discussion.

Table 1: Results for selected feed compositions and temperatures in Example 1.

\begin{tabular}{ccccc}
\multicolumn{2}{c}{ Feed } & \multicolumn{3}{c}{ Stationary } \\
$x_{0,1}$ & $T(\mathrm{~K})$ & $x_{1}$ & $\theta$ & $\widetilde{D}$ \\
\hline \multirow{3}{*}{0.6233} & 325.62 & 0.6233 & 0 & 0 \\
& & 0.4678 & 1 & -0.01439 \\
& & 0.2923 & 0 & -0.006359 \\
& & 0.8551 & 0 & -0.004804 \\
0.6233 & 325.243 & 0.6233 & 0 & 0 \\
& & 0.4684 & 1 & 0 \\
& & 0.2914 & 0 & -0.006428 \\
& & 0.8559 & 0 & -0.004878 \\
0.85822 & 325.243 & 0.85822 & 0 & 0 \\
& & 0.29703 & 0 & 0 \\
& & 0.4691 & 1 & 0.005939 \\
& & 0.6125 & 0 & 0.005537 \\
\hline
\end{tabular}

are many experimental liquid-phase points seen within this range. These discrepancies between model and experiment are plainly due to the fact that Uusi-Kyyny et al. (2004)'s model predicts a liquid-liquid split, while experimentally this apparently does not occur. The prediction of a liquidliquid split is due solely to the liquid-phase model used, and so it is clear that the NRTL model parameters given by Uusi-Kyyny et al. (2004) are poor. So how did Uusi-Kyyny et al. (2004) get this problem so badly wrong? First, in their parameter estimation procedure, they apparently fit the experimental data to an unstable solution of the phase split problem. Second, they then failed to realize that their model was incorrect because they solved subsequent phase equilibrium problems incorrectly, failing to find the stable 


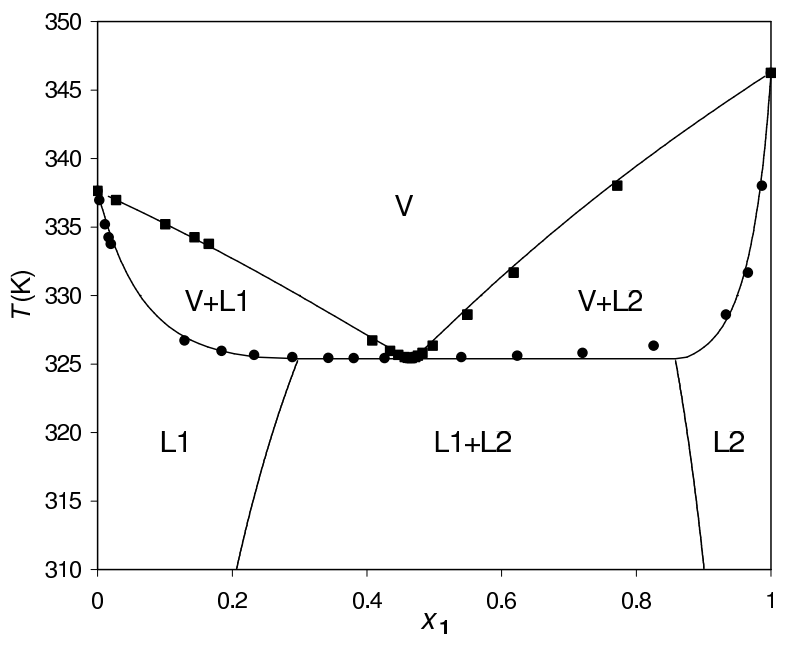

Figure 7: Phase diagram computed from Uusi-Kyyny et al. (2004)'s model in Example 1, along with their experimental data $(\boldsymbol{\square}=$ vapor; $\bullet=$ liquid $)$. See text for discussion.

solution. The combination of these two mistakes caused Uusi-Kyyny et al. (2004) to be misled into thinking they had successfully modeled their experimental results, when in fact they had not. Proper use of a reliable method for phase stability analysis would prevent this situation from occurring. Finally, note that these results do not necessarily mean that NRTL is a poor choice of model for the liquid, only that the parameters were poorly determined.

\section{$5.2 \quad$ Example 2}

In this example, we consider the binary mixture of dichlorodifluoromethane (CFC-12) (component 1) and hydrogen fluoride (component 2) at $T=303.15 \mathrm{~K}$. Again we consider an asymmetric model. The liquid-phase model is NRTL, as described above. The vapor-phase model is the Peng-Robinson (PR) EOS. The PR EOS is given by

$$
f(\boldsymbol{x}, v)=P-\frac{R T}{v-b}+\frac{a}{v(v+b)+b(v-b)}=0 .
$$

Here $a$ and $b$ are functions of $\boldsymbol{x}$ according to the mixing rules given above, and the pure component $a_{i}$ and $b_{i}$ are determined from

$$
\begin{gathered}
b_{i}=0.07780 \frac{R T_{c i}}{P_{c i}} \\
a_{i}=0.45724 \alpha \frac{R^{2} T_{c i}^{2}}{P_{c i}} \\
\alpha=\left[1+\beta\left(1-\sqrt{T / T_{c i}}\right)\right]^{2} \\
\beta=0.37464+1.54226 \omega_{i}-0.26992 \omega_{i}^{2} .
\end{gathered}
$$

Values of the model parameters are given below. The mixture fugacity coefficients $\breve{\phi}_{i}(\boldsymbol{x}, v)$ are determined (Prausnitz et al., 1986) from the PR EOS model, using the rela- tionship

$$
\begin{gathered}
\ln \breve{\phi}_{i}(\boldsymbol{x}, v)=\frac{b_{i}}{b}\left(\frac{P v}{R T}-1\right)-\ln \frac{P(v-b)}{R T} \\
-\frac{a}{2 \sqrt{2} b R T}\left[\frac{2 \sum_{j=1}^{n} x_{j} a_{j i}}{a}-\frac{b_{i}}{b}\right] \ln \frac{v+(1+\sqrt{2}) b}{v+(1-\sqrt{2}) b} .
\end{gathered}
$$

For this problem, the first-order optimality conditions, i.e., the equations solved using the interval-Newton approach, are again given by Eqs. (15)-(18).

Kang (1998) did measurements and modeling of the phase equilibrium for this system. These measurements of equilibrium pressure and average liquid-phase composition show that this system exhibits a maximum-pressure heterogeneous azeotrope (VLLE line), and Kang (1998) presents a model which appears to provide a good prediction of the pressure and the liquid-liquid phase split at the azeotrope. Kang (1998)'s model uses NRTL for the liquid phase. For the vapor phase, Kang (1998)'s base model is the PR EOS, but there is an additional contribution to the EOS to account for the association of HF molecules. For the computations done here, we will use the same liquidphase model (NRTL) as Kang (1998), but for the vapor phase, we will use the base PR model only, without the association terms. Thus we would expect our calculations to match liquid-liquid phase split results computed by Kang (1998), but not to match Kang (1998)'s results for vaporphase compositions or equilibrium pressures.

For the NRTL model, the parameters obtained by Kang (1998) are $A_{12}=1595.631 \mathrm{cal} / \mathrm{mol}$ and $A_{21}=1701.751$ $\mathrm{cal} / \mathrm{mol}$, with $\alpha_{12}=\alpha_{21}=0.425$. For the PR EOS, the pure component properties used are $T_{c 1}=385.0 \mathrm{~K}, P_{c 1}$ $=4129 \mathrm{kPa}, \omega_{1}=0.179, T_{c 2}=461.0 \mathrm{~K}, P_{c 2}=6480 \mathrm{kPa}$, and $\omega_{2}=0.372$, and the binary interaction parameter used is $k_{12}=0$. At the system temperature of $303.15 \mathrm{~K}$, the pure component vapor pressure values used by Kang (1998) are $P_{1}^{\text {sat }}=742.73 \mathrm{kPa}$ and $P_{2}^{\text {sat }}=144.0 \mathrm{kPa}$. Liquid molar volumes were taken to be $v_{1}^{\mathrm{L}}=95.804 \mathrm{~cm}^{3} / \mathrm{mol}$ and $v_{2}^{\mathrm{L}}=14.9 \mathrm{~cm}^{3} / \mathrm{mol}$, from the modified Rackett equation with parameters given by Kang (1998).

Kang (1998) computes a maximum-pressure heterogeneous azeotrope (VLLE line) at about $868 \mathrm{kPa}$, with the liquid splitting into one liquid phase with composition $x_{1} \approx$ 0.06 and other liquid phase with composition $x_{1} \approx 0.90$ (values estimated from a plot). Since the liquid-phase model (NRTL) is pressure-independent, the same liquidliquid split should be observed for pressures above the maximum-pressure heterogeneous azeotrope, where there should be liquid-liquid equilibrium only (no vapor). As an initial test point for our phase stability analysis procedure, we chose a pressure $(905 \mathrm{kPa})$ somewhat above the heterogeneous azeotrope, and a composition $\left(x_{0,1}=0.54\right)$ in the middle of the presumed two-phase (liquid-liquid) region. Results of applying phase stability analysis for this point are shown as the first feed in Table 2. This shows that actually this test phase is a stable liquid (single phase), and not in a two-phase region as Kang (1998)'s computations would indicate. Further analysis shows that there 
is a solution to the phase split problem with $x_{1}=0.0652$ for the first liquid phase and $x_{1}=0.8993$ for the second liquid phase. This is clearly the phase split found by Kang (1998) for the heterogeneous azeotrope. Phase stability analysis shows, however, that this is not a stable liquidliquid phase split, as indicated in Table 2 (second feed) and in the PTPD plot in Fig. 8. In fact, when Kang (1998)'s model is correctly solved, by use of the reliable phase stability analysis procedure given here, there are two VLLE lines (one a heterogeneous azeotrope) found. At the heterogeneous azeotrope and pressures above it, the liquid splits into one liquid phase with $x_{1}=0.5566$ and another liquid phase with $x_{1}=0.9013$. At the other VLLE line and pressures above it, the liquid splits into one liquid phase with $x_{1}=0.0647$ and another liquid phase with $x_{1}$ $=0.5360$. Both these phase splits were validated using phase stability analysis, as indicated in Table 2 (third and fourth feed) for trial points (905 kPa) somewhat above the VLLE pressures. Since this type of phase behavior is not observed experimentally, and the prediction of a liquidliquid split is due solely to the liquid-phase model used, it is apparent that the NRTL parameters determined by Kang (1998) are not appropriate. In a situation similar to that seen in the previous example, Kang (1998) apparently fit the experimental data to an unstable solution of the phase split problem, and then failed to recognize this because subsequent phase split calculations were done incorrectly (without correct phase stability analysis). This misled Kang (1998) into thinking that his NRTL parameters were reasonable.

\subsection{Example 3}

In this example, we focus on an approach for avoiding the sort of difficulties seen in the previous two examples. There are many good routines available for computing phase equi-

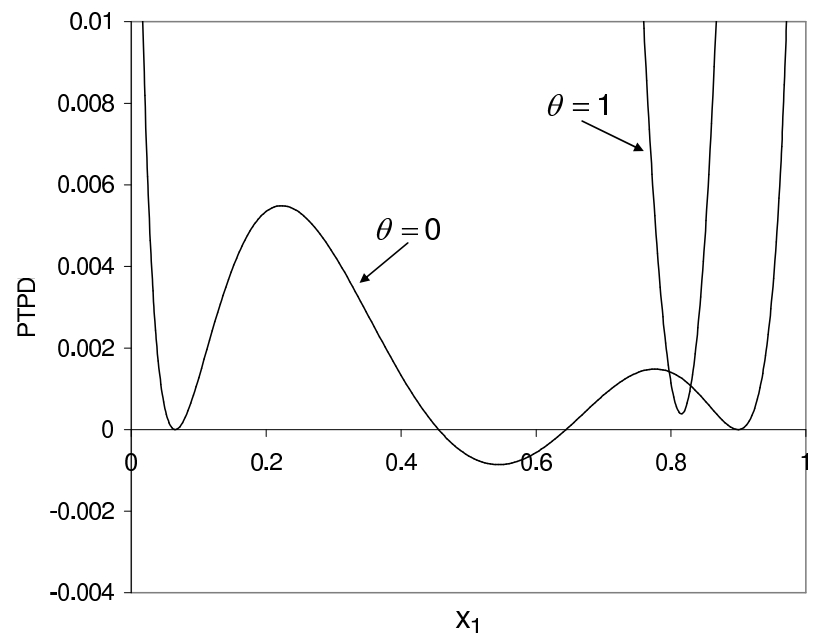

Figure 8: Plot of pseudo tangent plane distance (PTPD) function in Example 2 for liquid-liquid phase equilibrium computed by Kang (1998). This is not a stable equilibrium state. See text for discussion.
Table 2: Results for selected feed compositions and pressures in Example 2.

\begin{tabular}{|c|c|c|c|c|}
\hline \multicolumn{2}{|c|}{ Feed } & \multicolumn{3}{|c|}{ Stationary Points } \\
\hline$x_{0,1}$ & $P(\mathrm{kPa})$ & $x_{1}$ & $\theta$ & $\widetilde{D}$ \\
\hline \multirow[t]{6}{*}{0.54} & 905.0 & 0.54 & 0 & 0 \\
\hline & & 0.8151 & 1 & 0.001293 \\
\hline & & 0.2247 & 0 & 0.00604 \\
\hline & & 0.0649 & 0 & 0.0003998 \\
\hline & & 0.7796 & 0 & 0.002569 \\
\hline & & 0.8985 & 0 & 0.001201 \\
\hline \multirow[t]{6}{*}{0.0652055} & 905.0 & 0.0652 & 0 & 0 \\
\hline & & 0.8993 & 0 & 0 \\
\hline & & 0.8152 & 1 & 0.0001724 \\
\hline & & 0.2228 & 0 & 0.005488 \\
\hline & & 0.5446 & 0 & -0.0008581 \\
\hline & & 0.7762 & 0 & 0.001485 \\
\hline \multirow[t]{6}{*}{0.5566258} & 905.0 & 0.5566 & 0 & 0 \\
\hline & & 0.9013 & 0 & 0 \\
\hline & & 0.8156 & 1 & 0.0003807 \\
\hline & & 0.2181 & 0 & 0.007156 \\
\hline & & 0.0659 & 0 & 0.002048 \\
\hline & & 0.7672 & 0 & 0.0018 \\
\hline \multirow[t]{6}{*}{0.0646917} & 905.0 & 0.0647 & 0 & 0 \\
\hline & & 0.5360 & 0 & 0 \\
\hline & & 0.8150 & 1 & 0.001527 \\
\hline & & 0.2264 & 0 & 0.005776 \\
\hline & & 0.7826 & 0 & 0.002775 \\
\hline & & 0.8978 & 0 & 0.001505 \\
\hline
\end{tabular}

librium. These routines are often very reliable, but offer no guarantee of reliability and so fail on occasion to provide the correct results. What is needed is a way to validate the results of such routines when they are correct, and to provide feedback in the occasional case for which the results are incorrect. We demonstrate here a strategy for doing this, based on the use of the IN/GB approach for phase stability analysis described above. In particular, we adopt the standard code CHASEOS for computing chemical and phase equilibrium from cubic EOS models (e.g., SRK and PR), and show how its results can be validated, with corrective feedback as needed, using the routine INTSTAB, an implementation of the interval-based technique for phase stability analysis given above. CHASEOS implements the algorithm of Castier et al. (1989), an extension of the techniques of Michelsen (1982a,b) and Myers and Myers (1986). This is a well-regarded technique and is generally very reliable.

When the code CHASEOS returns a phase equilibrium result, there is no guarantee that it is correct. Thus, the result needs to be validated. To do this, the composition 
of one of the phases (or of the only phase if it is a singlephase result) is passed to INTSTAB for validation using phase stability analysis. If INTSTAB determines that the system is stable, then this validates that the result found by CHASEOS is indeed correct. If INTSTAB determines that the system is not stable, then this indicates that the results returned by CHASEOS are incorrect. In this case, the stationary point corresponding to the global minimum in $D$ will have a negative value of $D$. The composition at this stationary point is then returned to CHASEOS, where a new phase is added, and the composition at that stationary point is used in reinitializing before performing another phase split calculation. This process appears to the CHASEOS code just as if it were a stationary point with negative $D$ value found by its own stability analysis routine. CHASEOS is then executed until a new result is returned for validation by INTSTAB. This type of twostage strategy in which phase split computations (local minimization of the total Gibbs energy) alternates with phase stability analysis (global optimality check) can be shown (e.g., McKinnon et al., 1996) to converge in a finite number of steps to the equilibrium state (global minimum of the total Gibbs energy) provided that a deterministic procedure is used to globally minimize the tangent plane distance function in doing phase stability tests. In fact, CHASEOS already uses such a two-stage strategy (Michelsen, 1982a,b) internally, but the procedure used to test phase stability is not deterministic and may fail to give the correct result. This validated version of CHASEOS, obtained by interfacing CHASEOS and INTSTAB, is referred to as V-CHASEOS (Burgos-Solórzano et al., 2004). This validated computing approach could be used in connection with many other codes for phase equilibrium as well. If validation alone is desired, then the phase equilibrium code can be treated simply as a black box. If corrective feedback is also desired, then access to the source code is required, so that feedback can be inserted at the proper point in the code.

To demonstrate the use of V-CHASEOS, we will consider the mixture of acetic acid, ethanol, water, ethyl acetate and $\mathrm{CO}_{2}$ at $T=60 \mathrm{C}$ and $P=57.8 \mathrm{~atm}$. This problem arises in studying the esterification of acetic acid with ethanol using supercritical $\mathrm{CO}_{2}$ as a solvent:

$$
\text { acetic acid }+ \text { ethanol } \rightleftarrows \text { ethyl acetate }+ \text { water }
$$

This is a reactive system, and in solving the phase split problem, both reaction and phase equilibrium need to be accounted for. The phase stability problem is the same as described above. In this example, a symmetric model is used, with all phases (vapor or liquid) modeled using the PR EOS, as given by Eq. (20). All model parameters for this problem are given by Burgos-Solórzano et al. (2004); this is the third of three models they test for this system.

When stability analysis is done, the optimization problem to be solved for this symmetric model is given by Eq. (3). For this problem, the first-order optimality conditions, i.e., the equations solved using the interval-Newton
Table 3: Summary of steps done in application of $\mathrm{V}$ CHASEOS in Example 3.

\begin{tabular}{ll} 
Step & Result \\
\hline CHASEOS & Vapor + Liquid \\
INTSTAB & Not stable \\
CHASEOS (repeat 1) & Liquid + Liquid \\
INTSTAB (repeat 1) & Not stable \\
CHASEOS (repeat 2) & Vapor + Liquid + Liquid \\
INTSTAB (repeat 2) & Stable \\
\hline
\end{tabular}

approach, are

$$
\begin{gathered}
\frac{\partial D}{\partial x_{i}}-\frac{\partial D}{\partial x_{n}}=0, \quad i=1, \ldots, n-1 \\
1-\sum_{i=1}^{n} x_{i}=0 \\
f(\boldsymbol{x}, v)=0 .
\end{gathered}
$$

V-CHASEOS was applied for a feed containing $3.64 \mathrm{~mol}$ of ethanol, $3.64 \mathrm{~mol}$ of acetic acid and $2.72 \mathrm{~mol}$ of $\mathrm{CO}_{2}$. A summary of the steps taken in V-CHASEOS on this problem is shown in Table 3. The initial application of CHASEOS to this feed resulted in the computation of a vapor-liquid equilibrium. However, when INTSTAB attempted to validate this result, it was found to be not stable. Thus, without any validation step, the standard code CHASEOS would have returned an incorrect result, containing one liquid and one vapor phase. After corrective feedback from INTSTAB, CHASEOS now computes a liquid-liquid equilibrium. Again, when INTSTAB attempted to validate this result, it was found to be not stable. After another corrective feedback from INTSTAB, CHASEOS now computes vapor-liquid-liquid equilibrium, a result that is validated by INTSTAB. Thus, the correct validated result from V-CHASEOS is that there are two liquid phases and one vapor phase, not a single liquid phase as CHASEOS alone would have determined.

\section{CONCLUDING REMARKS}

The computation of phase equilibrium is a very challenging problem. There have been proposed a very large number and wide variety of problem formulations and numerical solution procedures, involving both direct optimization and the solution of equivalent nonlinear equation systems. Still, with very few exceptions, these methods may fail to solve the phase equilibrium problem correctly in some cases. It is, unfortunately, not difficult to find examples in the literature of phase equilibrium problems that have been solved incorrectly. We have described here a deterministic method, based on interval analysis, that provides a mathe- 
matical and computational guarantee that the phase equilibrium problem has been correctly solved, and have highlighted recent results using this approach.

The use of interval methods to validate results from a standard phase equilibrium code has also been demonstrated, in connection with the standalone code CHASEOS. This approach to validated computing could also be used for phase equilibrium computations in the context of a process simulator. In this case, since the equilibrium computation may be nested inside some other iterative calculation, it is probably not desirable to invoke the validation procedure every time an equilibrium computation is done. Instead, the validation step should be applied only after the entire simulation is complete. The validation comes at the cost of additional computing time. Thus a modeler may need to consider the trade off between the additional computing time and the risk of getting the wrong answer to a phase equilibrium problem. Certainly, for mission critical situations, the additional computing expense is well spent.

\section{ACKNOWLEDGEMENTS}

This work was supported in part by The State of Indiana 21st Century Fund under Grant 031500-0077, by the donors of The Petroleum Research Fund, administered by the ACS, under Grant 35979-AC9, and by the U. S. Department of Energy under Grant DE-FG02-05CH11294.

\section{REFERENCES}

Adjiman, C. S., Androulakis, I. P., Floudas, C. A., and Neumaier, A. (1998). A global optimization method, $\alpha \mathrm{BB}$, for general twice-differentiable NLPs-I. Theoretical advances. Comput. Chem. Eng., 22:1137-1158.

Baker, L. E., Pierce, A. C., and Luks, K. D. (1982). Gibbs energy analysis of phase equilibria. Soc. Petrol. Engrs. J., 22:731-742.

Burgos-Solórzano, G. I., Brennecke, J. F., and Stadtherr, M. A. (2004). Validated computing approach for highpressure chemical and multiphase equilibrium. Fluid Phase Equilib., 219:245-255.

Castier, M., Rasmussen, P., and Fredenslund, A. (1989). Calculation of simultaneous chemical and phase equilibria in nonideal systems. Chem. Eng. Sci., 44:237-248.

Falk, J. E. and Soland, R. M. (1969). An algorithm for separable nonconvex programming problems. Manage. Sci., 15:550-569.

Floudas, C. A. and Visweswaran, V. (1990). A global optimization algorithm (GOP) for certain classes of nonconvex NLPs: I. Theory. Comput. Chem. Eng., 14:13971417 .
Floudas, C. A. and Visweswaran, V. (1993). A primalrelaxed dual global optimization approach. J. Optim. Theory Appl., 78:187-225.

Gau, C.-Y. and Stadtherr, M. A. (2002). New interval methodologies for reliable chemical process modeling. Comput. Chem. Eng., 26:827-840.

Hansen, E. and Walster, G. W. (2004). Global Optimization Using Interval Analysis. Marcel Dekker, New York.

Harding, S. T. and Floudas, C. A. (2000). Phase stability with cubic equations of state: Global optimization approach. AIChE J., 46:1422-1440.

Hua, J. Z., Brennecke, J. F., and Stadtherr, M. A. (1996a). Reliable phase stability analysis for cubic equation of state models. Comput. Chem. Eng., 20:S395-S400.

Hua, J. Z., Brennecke, J. F., and Stadtherr, M. A. (1996b). Reliable prediction of phase stability using an intervalNewton method. Fluid Phase Equilib., 116:52-59.

Hua, J. Z., Brennecke, J. F., and Stadtherr, M. A. (1998). Enhanced interval analysis for phase stability: $\mathrm{Cu}$ bic equation of state models. Ind. Eng. Chem. Res., 37:1519-1527.

Jaulin, L., Kieffer, M., Didrit, O., and É Walter (2001). Applied Interval Analysis. Springer-Verlag, London.

Kang, Y. W. (1998). Vapor-liquid equilibria for the systems difluoromethane + hydrogen fluoride, dichlorodifluoromethane + hydrogen fluoride, and chlorine + hydrogen fluoride. J. Chem. Eng. Data, 43:13-16.

Kearfott, R. B. (1996). Rigorous Global Search: Continuous Problems. Kluwer Academic Publishers, Dordrecht, The Netherlands.

Lin, Y. and Stadtherr, M. A. (2004a). Advances in interval methods for deterministic global optimization in chemical engineering. J. Global Optim., 29:281-296.

Lin, Y. and Stadtherr, M. A. (2004b). LP strategy for the interval-Newton method in deterministic global optimization. Ind. Eng. Chem. Res., 43:3741-3749.

Makino, K. and Berz, M. (1999). Efficient control of the dependency problem based on Taylor model methods. Reliab. Comput., 5:3-12.

Makino, K. and Berz, M. (2003). Taylor models and other validated functional inclusion methods. Int. J. Pure Appl. Math., 4:379-456.

McDonald, C. M. and Floudas, C. A. (1995a). Global optimization and analysis for the Gibbs free energy function using the UNIFAC, Wilson, and ASOG equations. Ind. Eng. Chem. Res., 34:1674-1687. 
McDonald, C. M. and Floudas, C. A. (1995b). Global optimization for the phase and chemical equilibrium problem: Application to the NRTL equation. Comput. Chem. Eng., 19:1111-1139.

McDonald, C. M. and Floudas, C. A. (1995c). Global optimization for the phase stability problem. AIChE J., 41:1798-1814.

McDonald, C. M. and Floudas, C. A. (1997). GLOPEQ: A new computational tool for the phase and chemical equilibrium problem. Comput. Chem. Eng., 21:1-23.

McKinnon, K. I. M., Millar, C. G., and Mongeau, M. (1996). Global optimization for the chemical and phase equilibrium problem using interval analysis. In Floudas, C. A. and Pardalos, P. M., editors, State of the Art in Global Optimization: Computational Methods and Applications, pages 365-382, Dordrecht, The Netherlands. Kluwer Academic Publishers.

Michelsen, M. L. (1982a). The isothermal flash problem. Part I: Stability. Fluid Phase Equilib., 9:1-19.

Michelsen, M. L. (1982b). The isothermal flash problem. Part II: Phase-split calculation. Fluid Phase Equilib., 9:21-40.

Moore, R. E. (1966). Interval Analysis. Prentice-Hall, Englewood Cliffs, NJ.

Myers, A. K. and Myers, A. L. (1986). Numerical solution of chemical equilibria with simultaneous reactions. $J$. Chem. Phys., 84:5787-5795.

Nagarajan, N. R., Cullick, A. S., and Griewank, A. (1991). New strategy for phase equilibrium and critical point calculations by thermodynamic energy analysis. Part I. Stability analysis and flash. Fluid Phase Equilib., 62:191-210.

Neumaier, A. (1990). Interval Methods for Systems of Equations. Cambridge University Press, Cambridge, UK.

Neumaier, A. (2003). Taylor forms - Use and limits. Reliab. Comput., 9:43-79.

Prausnitz, J. M., Lichtenthaler, R. N., and de Azevedo, E. G. (1986). Molecular Thermodynamics of Fluid-Phase Equilibria. Prentice Hall, Englewood Cliffs, NJ.

Rohn, J. and Kreinovich, V. (1995). Computing exact componentwise bounds on solution of linear systems with interval data is NP-hard. SIAM J. Matrix Anal. Appl., $16: 415-420$.

Schnepper, C. A. and Stadtherr, M. A. (1996). Robust process simulation using interval methods. Comput. Chem. Eng., 20:187-199.

Seider, W. D. and Widagdo, S. (1996). Multiphase equilibria of reactive systems. Fluid Phase Equilib., 123:283303.
Stadtherr, M. A., Schnepper, C. A., and Brennecke, J. F. (1995). Robust phase stability analysis using interval methods. AIChE Symp. Ser., 91(304):356-359.

Tessier, S. R., Brennecke, J. F., and Stadtherr, M. A. (2000). Reliable phase stability analysis for excess Gibbs energy models. Chem. Eng. Sci., 55:1785-1796.

Uusi-Kyyny, P., Pokki, J.-P., Kim, Y., and Aittamaa, J. (2004). Isobaric vapor liquid equilibrium for 2,3-dimethyl-2-butene + methanol, + ethanol, +2 propanol, or +2 -butanol at atmospheric pressure. $J$. Chem. Eng. Data, 49:251-255.

Xu, G., Brennecke, J. F., and Stadtherr, M. A. (2002). Reliable computation of phase stability and equilibrium from the SAFT equation of state. Ind. Eng. Chem. Res., 41:938-952.

Xu, G., Haynes, W. D., and Stadtherr, M. A. (2005). Reliable phase stability analysis for asymmetric models. Fluid Phase Equilib., 235:152-165. 